\title{
The Effects of Cell Phone Radiation on the Human Body: An Overview
}

\author{
Jai Om
}

\begin{abstract}
In this research article, an overview is provided of all the plausible effects that cell phone radiation might have on a human body. The number of cell of regular cell phone users has exploded around the world, and cell phones have become an integral part of our lives in the recent past. Therefore, this paper strives to answers public questions regarding the safety of using cell phones. It analyses previous research that has been done in the field to provide concise answers. The article also supports the motion that cell phones can be harmful in the long run; however, there is no conclusive evidence. The future research should be made more streamlined, and focus on human body systems.
\end{abstract}

Index Terms-Cell phone, radiation, effects, cancer, human body.

\section{INTRODUCTION}

$\mathbf{I}$ $\mathrm{N}$ the modern era of science and development, there has been a major revolution in the field of communication. The number of cell phones has skyrocketed in the recent past [1]. Rough estimates show that there are about 1.6 billion people world-wide who use cell phones on a regular basis [2]. Cell phone technology generally uses Radio-frequency, more commonly referred to as the RF, for the data transmission [3]. The radiation is measured in terms of frequency, in units known as hertz $(\mathrm{Hz})$, and the wavelength of the radiation. Frequency is the number of complete cycles of the wave that pass per second through a fixed point. Communication through mobile phones comes under a global network known as the Global System for Mobile Communication, usually referred as GSM. Cell phones operate over a range of frequencies, such as $850 \mathrm{MHz}, 900 \mathrm{MHz}$, and $1800 \mathrm{MHz}$ in the GSM network, and therefore the prefix GSM is added to these frequencies, to indicate that these frequencies are used for cellular communication [2]. To add on, it is important to understand the basic concepts of energy associated with the RF waves, as this energy is the main cause that leads to different effects on the human body. RF energy is a part of the electromagnetic radiation, and the electromagnetic radiation can be further separated into two categories which are ionizing and non-ionizing. Examples of ionizing types of radiation are cosmic rays and X-rays; meanwhile radio-frequency is an example of the non-ionizing type.

This massive increase in the regular users of mobile phones has led to many experts from around the globe raising questions regarding the safety of cell phone usage. Research has been constantly going on in order to investigate if cell phones have any negative health implications, or if radiation from cell

DOI: http://dx.doi.org/10.24018/ejers.2019.4.3.1192

Published on March 26, 2019.

Jai Om is with the Department of EECE, University of Pretoria, Pretoria, South Africa (e-mail: jaiom1998@gmail.com). phone antennas can cause serious disorders among the human population. Many world renowned researchers and scientists have carried out experiments, and published results, in this field to find out if cell phone radiation can actually increase the risk of developing cancer, or any other life threatening conditions.

The main focus of majority of the experiments has been to investigate the heating effect caused by the cell phone radiation. It has been widely acknowledged that continuous usage of cell phone for an extended period of time leads to an increase in the temperature in the head region, and this increase in temperature has been linked with structural damage to the Blood Brain Barrier [4]. Many published articles critically analyse the results that were produced by other research groups, in order to fully validate the produced results [5]. Some experiments have found astonishing results, where the radiation from the cell phone towers and antennas has actually proved beneficial in certain situations [1]. These results have not been accepted across the world, and are being critically analysed. One of the major drawbacks in this field of research has been the inability to reproduce results that were produced by a separate research group. The biggest reason for this inability is the sheer amount of variables involved in this experiment [6]. Every individual reacts differently to the heating stimulus produced as a result of long-term exposure to cell phone. Therefore, the results obtained from a certain research cannot be generalised. The majority of the researchers also agree that there are certain aspects of the cell phone radiation that are not yet well understood, and therefore the amount of confidence in the findings of research is quite low among the world wide cell phone users.

This paper provides a summary of various researches that has taken place in this field, and provides a brief conclusion based on the results of these experiments. It is important to note that this article does not intend to support any of the claims regarding the cell phone radiation, as no active experiment has been undertaken by the researcher. It is only a concise collection of various research studies from around the world, to be presented in a simple fashion for the end-users.

\section{POSSIBLE EFFECTS OF CELL PHONES}

\section{A. The Heating Effect caused by the Cell phone radiation [2]}

It has been acknowledged by many previous researchers that cell phone radiation causes a heating effect in the human systems. The radiation from the cell phone can be absorbed by the somatic tissues close to the area of exposure. There is a metabolic function in our bodies that control the regulation of the body temperature, known as the thermoregulatory system 
[2]. However, it is possible that with time this system degrades due to continuous long term exposure to cell phone radiation. In the research that was carried out to investigate the heating effect of the electromagnetic radiation in the head region provided definite results.

The researchers investigated the effect caused by two different types of cell phones, namely GSM 900 and GSM 1800. The experiments produced some interesting results. It clearly showed that usage of cell phones increased the temperature of the head region by a significant amount. Cell phones of $900 \mathrm{MHz}$ caused a higher temperature, as compared to $1800 \mathrm{MHz}$; however the difference between the two frequencies is not significant. The usage of headphones and Bluetooth head devices do not increase the temperature in the head region.

\section{B. The Blood-Brain Barrier and its Permeability [4]}

Before the analyze the possible impacts of cell phone radiation on the Blood Brain Barrier, more commonly known as the $\mathrm{BBB}$, it is important to understand what exactly is the $\mathrm{BBB}$ and its main function.

The BBB is an integral part of our cerebral vascular system. It forms a lining around the brain, and consists of complex structures and proteins that control the movement of substances in and out of the brain. It plays the role of a barrier for foreign substances and prevents them from reaching the brain. It is a crucial component in keeping the brain safe from chemical and biological agents.

In some recent studies, researchers have found that radiation form the cell phone can really have a serious impact on the Blood Brain Barrier. The radiation can affect permeability of the barrier. It has been hypothesized that the permeability of the $\mathrm{BBB}$ can increase due to exposure to cell phone radiation for an extended period of time. This allows for more substances to reach the brain cells. And it can lead to many foreign substances attacking the brain.

However, it is important that there has been a counter argument for this scenario. Some research shows that the BBB comes back to its original state after some time, and therefore there is no long-lasting impact on the BBB.

\section{The Effect on Cancer Risk Factor}

This research article focuses on the risk factor of cancer that occurs as a result of cell phone radiation. In essence, it makes more sense to discuss the risk factor, because every individual is unique and their body reacts in a different way. Therefore, it is not possible to conclusively say that cell phones can cause cancer, as it is possible that they might not cause any form of cancer in some individuals despite of long-term usage. Meanwhile, risk factor can be analysed as a variable that is the same for everyone, and therefore it provides a better picture if cell phones can cause cancer in human bodies.

There is a lot of debate in this field, as various contradictory results have been published regarding the link between cancer and cell phone usage. In a research article published in 2008 [6], it showed that the risk factor could be increased by as much as $470 \%$ of developing malignant brain tumours after a long-term usage of cell phone.

However, some other research contradicts such high numbers. One research paper [5] mentioned the risk factor of brain tumours was at $40 \%$. Another mentioned article shows a risk factor is increased by $270 \%$ for regular users of cell phones. The third paper showed an increase factor ranging from $10 \%$ to $300 \%$ for neuroma in the acoustic region, which is directly linked to long-term exposure to cell phones [5].

It is evident that these numbers do not provide a generalised trend. There is an obvious increase in the risk factor when cell phone usage has been for a long period of time, such as 10-15 years. However, there is no concrete evidence that cell phone radiation can cause cancer among the human beings.

It is also important to note that many researchers agree on one aspect of cell phone usage. Use of cell phones for a shorter period of time, such as 5 years, does not cause any cancer or significant increase in the risk factor [3].

\section{Effects on the DNA and Genetic Structure}

Research has also been carried out in this field to investigate the impact of cell phone usage on the genetic structure of the exposed somatic cells.

According to a clinical experiment [7], the effects of GSM 900 cell phone radiation on the chromosomal structure were observed. The researchers tried to see if there was any effect on the DNA due to exposure to radiation. The researchers found very definite results from their experiments. It was clear from the experiment that there was no effect on the genetic structure due to the exposure to the radiation. There were no traces of aneuploidy in any of the chromosomes. To conclude, the researchers claimed that there was no direct effect of radiation at that particular frequency, to the human body cells.

In another conference, [8] researchers claimed that there was a possibility that cell phone radiation might have negative implications on the DNA structure, related to the increased risk factor of developing cancer.

\section{E. Other Negative Implications}

Some research has also shown that cell phones have other negative implications on our bodies.

Some of the other common disorders that have been linked with cell phones are Alzheimers disease, headaches, drowsiness, reduced functionality of the immune systems, and many more [1][6]. However, it is important to note that the degree of these disorders depend on various factors, such as the strength of the radiation, different frequency levels might have varied implications, the nature of modulation, polarization levels, and the duration of exposure in relation to the strength of radiation might also lead to a range of different conditions. All of these variables are difficult to control in experimental setups, and therefore results from experimental studies might not truly reflect the extent of effects that cell phone radiation might have on a human body.

Some medical studies have also shown that the negative effects also vary based on the human body. The dielectric field inside organic tissues can react differently with different 
forms of radiation, and therefore has a massive impact on the effects of radiation [1]. The human characteristics are unique to everybody and therefore it is impossible to replicate all the possible impacts in an experiment, but including the human internal fields as a variable would lead to more accurate results and help to identify more negative implications of the cell phone radiation.

\section{F. Some Possible Positive Effects}

Some researchers have also claimed that there is a possibility that cell phone usage might prove beneficial in some cases. There is not a lot of evidence for this claim, and many endusers do not know about this aspect of the research; however it could be an important breakthrough for medical science to be able to use cellphone radiation in a controlled environment to our benefit.

One such case has been the Blood Brain Barrier. Some experts argue that the increase in the permeability of the BBB can be used for medical purposes. BBB acts a protective barrier for the brain against foreign substances and prevents them from reaching the brain through blood. Conventional medication to treat brain tumors that is administered intravascular might not be very effective as some portion of the drug gets rejected by the $\mathrm{BBB}$ and it never reaches the brain tissues. However, some studies show that if the BBB gets disrupted in a controlled manner, then the medication would be more effective for treating brain tumors as sufficient quantities would reach the desired tissues. Hence, some researchers claim that it might be possible to carry out chemotherapy of the brain more effectively after exposing the BBB to radiation from the cell phone as more chemical agents will be able to reach the brain, making the process more beneficial for the patient [4].

It has also been hypothesized that radiation can also be used in healing processes, and regeneration of damaged body parts, such as limbs, or organs [1]. However, there is no concrete medical evidence to support this hypothesis. The basis of this theory is that radiation from this cell phone radiation can lead to an increase in the metabolic rate of the human cells, which can then be controlled for the healing process and the regeneration of desired human tissues.

\section{CONCLUSION}

From all the results that have been published over the years, it is evident that there is no concrete evidence that usage of mobile phones can lead to cancer. It may have some side-effects, such as headache [1]; however, these effects are not long-lasting and are generally not very serious. There is no conclusive epidemiological proof that cell phones can cause cancer or other life-threatening disorders [9]. Some research does show that exposure to cell phone radiation for an extended period of time can have a significant increase in the risk factor of developing cancer. Many researchers have also questioned this, and are trying to reproduce similar results to verify their validity. However, this data really puts things into perspective. It is possible that the risk factor published in the article might be inflated, but it might point out to a very negative possibility. Even if the usage of cell phones increases the risk factor by a minimal amount, it might have very serious global implications. The number of cell phone users has increased exponentially in the recent times, and there are about 1.6 billion users [2]. Even a slight increase in the risk factor can put many millions of lives at risk. It is a very simple logical explanation: more the users, higher the possibility of health risks. Therefore, it is imperative to fully understand the effects of cell phone radiation on the human body because even if there is a slight increase in the risk factor, it means that lots of people are continuously exposing themselves to harmful radiation. It is also important that all the research findings are communicated well with the people all across the world, and not just in the more developed parts of the globe. It is extremely important to tell the people about all the possible effects of the phone on the body, so everyone has a chance to make a conscious decision about using cell phones over an extended period of time. Furthermore, if the users are well aware of all the possible implications, then it will be easier for them to decide for themselves if there is a need to limit their cell phone usage.

One of the major suggestions that can be given for future research is to find the clinical effects on the human body, because majority of the research is being done on animals [9]. And it is possible that the effects of cell phone radiation might be different on the human body. We have to wait for more clinical experiments, in order to come up with any concrete generalised trend [10]. In the meantime, it is crucial that cell phone users try to reduce their cell phone usage [9] to reduce the risk of developing life-threatening disorders because the negative effects from the cell phones are highly possible.

Adding on, it is also important to extend the research to cover all the different ways in which cell phones might affect the human body, including the psychological affects that cell phones cause. This is an age of social media, and almost everyone is connected to each other through the world of social websites. Therefore, this puts everyone at an added risk and dangers that the world of social media possesses. Some psychologists and medical experts have pointed out that the world of social media can lead to more stress and anxiety among some people due to various reasons, such as introvert personality. However, this is still a hypothesis in its initial stages, and the psychological effects have not been at the forefront of all the research regarding the effects of cell phone on the human body.

It is crucial to understand that all the effects of the cell phones have to be researched more critically, including the physical and the psychologically effects. Then, it is also imperative that all the results are shared with the public on a large scale so that everyone can be fully aware of what the scientists and medical researchers have discovered, and so that people can make conscious decisions concerning their decisions regarding the use of cell phones.

\section{ACKNOWLEDGMENT}

I would take this opportunity to first thank God for all his blessings.

I would like to thank the department of Electrical Electronic and Computer Engineering (EECE) for accepting me and 
giving me a chance to work in this field. I would also like to thank my lecturer Dr. H. Myburgh, who played an important role for this article. My parents, and my family, also supported me, and proved to be the best guides that I could have. I thank them for all their support and guidance. A special mention for my father, who agreed to help me financially for this article, and gave me all the help I needed. I would like mention the last words of gratitude for all my friends, who were there for me through thick and thin, and motivated me throughout this work.

\section{REFERENCES}

[1] E. Vinodha and S. Raghavan, "Possible effects of cell phone radiation: An overview paper," in 2015 2nd International Conference on Electronics and Communication Systems (ICECS), 2015, pp. 837-841.

[2] F. Kho, A. Joseph, K. Kipli, S. Sahrani, K. Lias, A. S. W. Marzuki, and D. A. A. Mat, "Electromagnetic radiation from mobile phone near ear-skull region," in 2010 International Conference on Computer and Communication Engineering (ICCCE), 2010, pp. 1-5.

[3] A. Ahlbom, M. Feychting, and S. Lnn, "Mobile phones and tumour risk: Interpretation of recent results," Radio Science Bulletin, USRI, no. 314, pp. 30-33, 2005.

[4] J. C. Lin, "The blood-brain barrier, cancer, cell phones, and microwave radiation," IEEE Antennas and Propagation Magazine, vol. 43, no. 3, pp. 141-143, 2001.

[5] J. C. Lin, "Are radio frequency or cell phone fields possibly carcinogenic to humans?" IEEE Microwave Magazine, vol. 13, no. 4, pp. 30-32, May 2012.

[6] R. Seetharaman, G. S. Uthayakumar, N. Gurusamy, and N. Kumaravel, "Mobile phone usage and cancer," in 2008 10th International Conference on Electromagnetic Interference and Compatibility, 2008, pp. 627-632.

[7] C. Yardin, Bourthoumieu, P. Leveque, and A. Collin, "Chromosomal studies of human amniotic cells exposed to gsm-900: Karyotyping and fish," in 2011 XXXth URSI General Assembly and Scientific Symposium, 2011, pp. 1-2.

[8] N. Kumar, "Health effects of non-ionized electromagnetic radiation," in 2008 International Conference on Recent Advances in Microwave Theory and Applications, 2008, p. 276.

[9] K. R. Foster and J. E. Moulder, "Are mobile phones safe?" IEEE Spectrum, vol. 37, no. 8, pp. 23-28, 2000.

[10] J. C. Lin, "Can cell phones promote brain tumors, the interphone study?" Antennas and Propagation Magazine, vol. 47, no. 2, pp. 137-138, 2005.

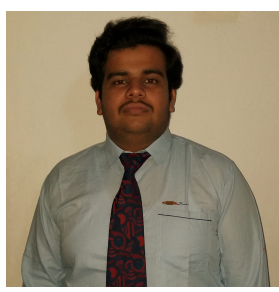

Jai Om: I am a Computer Engineering student in the Department of Electrical, Electronic and Computer Engineering; University of Pretoria, South Africa. I am intrigued by the field of hardware development and defense technology. My school results have been up to scratch, with a distinction average for all my university years. I also have a keen interest in community welfare, using engineering research and improvement of infrastructure. My ultimate goal is to pursue engineering research and design, and to encourage sustainable development in the field of

science and engineering. 